\section{Die Diagnostik von Allergien und Intoleranzreaktionen auf Hymenopterengifte mit Hilfe des
Zellantigenstimulationstests (CAST) Hymenopterengifte mit Hilfe des
Zellantigenstimulationstests (CAST)}

Zusammenfassung. Der Zellantigenstimulationstest (CAST) stellt ein neues Verfahren in der In-vitro-Diagnostik von allergischen Reaktionen dar. Dieser Test beruht auf der Stimulierung von Blutleukozyten mit anschließender Bestimmung der produzierten Sulfidoleukotriene $\mathrm{LTC}_{4}$ und deren Metaboliten $\mathrm{LTD}_{4}$ und $\mathrm{LTE}_{4}$. Die Sulfidoleukotriene werden mittels ELISA-Technik (Enzyme Linked Immunosorbent Assay) gemessen. 84 Patienten mit Verdacht auf Hymenopterengiftallergie wurden mit Hilfe des CAST, des CAP-Systems und der klassischen Hauttests untersucht. Beim vorliegenden Untersuchungsmaterial wurden allergische Reaktionen aller Schweregrade (Grad I-IV nach Mueller) diagnostiziert. Die allergologische Abklärung umfasste nach einer gründlichen Anamnese den Hauttest (Schwellenwertbestimmung bis $1 \mu \mathrm{g} / \mathrm{mL}$ ), die spezifische IgE-Antikörper-Bestimmung mit dem CAP-System und den zellulären Antigenstimulationstest. Der CAST wurde mit einer Konzentration von jeweils $0,2 \mu \mathrm{g} / \mathrm{mL}$ Bienen- und Wespengift (Apis mellifera et Vespula spec.) durchgeführt. Als Vergleich diente eine Kontrollgruppe von 10 nicht-allergischen CAP-negativen Personen. Zur Evaluation der diagnostischen Wertigkeiten des CAST wurden sowohl Vergleiche mit dem Hauttest als auch mit den Ergebnissen des CAP-Systems herangezogen. Die Sensitivität und Spezifität erreichten mit 93/67\% für Bienengift und 96/63\% für Wespengift sehr gute Werte und war den CAP-Ergebnissen überlegen. Durch Inkubation mit C 5a war es möglich, in einigen Fällen eine pseudoallergische Reaktion nachzuweisen.

Diagnosis of Allergic and Intolerance Reactions Against Hymenoptera Venoms by Means of the Cellular Antigen Stimulation Test (CAST). The Cellular Antigen Stimulation Test (CAST) is a new method of in-vitro-diagnosis of allergic reactions. The test is based on the prestimulation of blood leukocytes with cytokine IL-3 and the exposure to allergens. Mainly basophiles react by synthesizing sulfidoleukotrienes LTC $_{4}$ and its metabolites $\mathrm{LTD}_{4}$ and $\mathrm{LTE}_{4}$, which can be detected by ELISA technique. 84 patients with suspected allergy to hymenoptera venoms were investigated by means of the CAST, CAP, and the skin tests. Allergic reactions comprised all severity grades (I-IV ref. H. L. Mueller). The allergological workup included skin test (up to $1 \mu \mathrm{g} / \mathrm{mL}$ ), the measurement of specific IgE-antibodies by

Akt Dermatol 2001; 27: $223-229$

(c) Georg Thieme Verlag Stuttgart · New York ISSN 0340-2541

\author{
U.-C. Hipler ${ }^{1}$, G. Schlenvoigt ${ }^{2}$, A. Bauer ${ }^{1}$, P. Elsner ${ }^{1}$ \\ ${ }^{1}$ Klinik für Hautkrankheiten, Klinikum der Friedrich-Schiller- \\ Universität, Jena (Direktor: Prof. Dr. P. Elsner) \\ 2 Institut für Klinische Immunologie, Klinikum der Friedrich- \\ Schiller-Universität, Jena (Direktor: Prof. Dr. L. Jäger)
}

CAP system and Cellular Antigen Stimulation Test. The CAST was carried out with a concentration of $0.2 \mu \mathrm{g} / \mathrm{mL}$ bee and wasp venom (Apis mellifera, Vespula spec.), respectively. A control group of 10 non-allergic CAP-negative individuals was established. Diagnostic accuracy of CAST was evaluated by comparison with the skin tests and CAP. With bee venom a sensitivity and specificity of $93 / 67 \%$ was obtained, while wasp venom showed $96 / 63 \%$ sensitivity and specificity. In our investigations the CAST showed better results in comparison to CAP results. In some cases pseudoallergic reactions could be detected after incubation with $\mathrm{C} 5 \mathrm{a}$.

\section{Einleitung}

Unter epidemiologischer Sicht werden durch Hymenopterenstiche ausgelöste gesteigerte örtliche Reaktionen von $2-19 \%$, systemische Überempfindlichkeitsreaktionen von 0,8-5\% der Bevölkerung in Europa oder den USA angegeben [21]. Bei gleichzeitiger Berücksichtigung von Anamnese, Hauttestsoder RAST-Ergebnissen wurden Hinweise auf eine Sensibilisierung gegenüber Hymenopterengiften bei bis zu einem Viertel der Bevölkerung gefunden [17]. Ebenso wie bei anderen Allergien stehen bei allergischen Insektengiftreaktionen die In-vivo-Testungen und die In-vitro-Tests zur Verfügung. In Tab. 1 wird eine kurze Übersicht der möglichen Testverfahren gegeben.

Wichtige Aussagen über den Pathomechanismus kann man sehr gut mit Hilfe der In-vitro-Testverfahren erhalten. Man unterscheidet zwischen serologischen und zellulären Testverfahren. Zu den serologischen Tests gehört der Nachweis der spezifischen IgE-Antikörper gegen verschiedene Allergene mittels der RAST-, CAP-, Magic Lite- und Flüssigallergen-Technik. Abb. 1 gibt einen Überblick über die Spezifitätsdiagnostik und die Aktivitätsdiagnostik bei allergischen Erkrankungen [11].

Der zelluläre Antigenstimulationstest zählt zu den zellulären Tests in der Diagnostik von allergischen und pseudoallergischen Reaktionen und wurde von de Weck et al. [5,6] entwickelt. Die Methode basiert auf der Messung von Sulfidoleukotrienen, die durch Stimulation in Zellen produziert werden. Die Sulfidoleukotriene (sLT) LTC $_{4}$, LTE$_{4}$ und LTD $_{4}$ sind Produkte der Arachidonsäure, die durch 5-Lipoxygenase und Gluthation-STransferase umgewandelt wird (Abb. 2). 


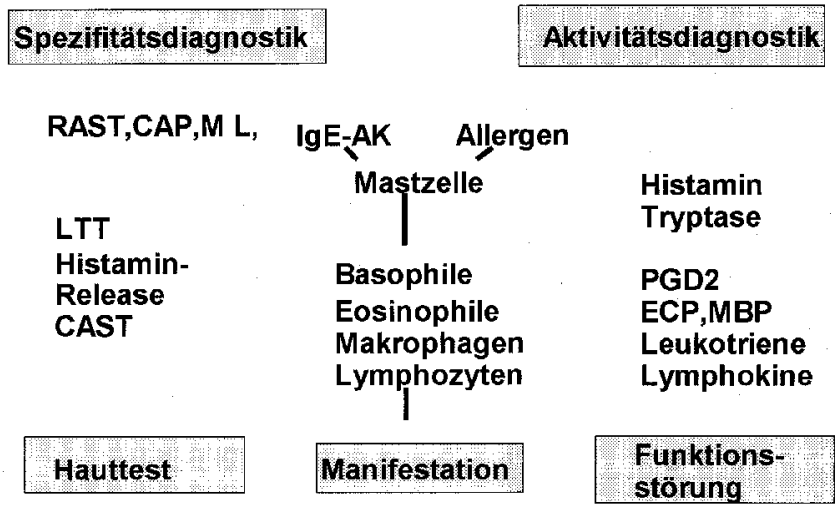

Abb.1 Möglichkeiten der allergologischen Diagnostik.

Das Zusammenfließen der vernetzten IgE-Moleküle bzw. Rezeptoren auf der Oberfläche der Zellen stellt das für die Sekretion notwendige Membransignal dar. Der Mechanismus des Übersetzungsprozesses ist noch unklar. Man diskutiert jedoch 3 Hauptmechanismen, die die Aktivierung und Degranulation der Mastzellen und Basophilen erklären [13]:

1. Methylierung von Phospholipiden durch Methyltransferasen

2. über ein GTP-bindendes stimulatorisches G-Protein, welches die Adenylatzyklase (AC) aktiviert

3. über Phospholipase $C$ und ein intermediäres GTP-bindendes Protein

Nach dem einen Schema aktiviert die $\mathrm{Fc}_{\varepsilon}$-Rezeptor Aggregation Methyltransferasen, die dann Membranphospholipide methylieren und Kalziumkanäle aktivieren. Nach dem zweiten Schema stimulieren die aggregierten IgE-Rezeptoren ein GTP-bindendes stimulatorisches Protein, das wiederum eine Adenylatcyclase aktiviert. Diese überführt dann ATP in zyklisches AMP. Der erhöhte Spiegel des zyklischen AMP aktiviert Proteinkinasen, die darauf eine Freisetzung von $\mathrm{Ca}^{2+}$-Ionen aus intrazytoplasmatischen Speichern bewirken. Nach dem dritten Schema aktivieren die aggregierten Rezeptoren die Phospholipase C, wahrscheinlich über ein intermediäres GTP-bindendes Protein. Die Phospholipase C spaltet Phosphatidylinositol und produziert zwei Second Messenger, das Phosphatidylinositol-4,5-biphosphat $\left(\mathrm{PIP}_{2}\right)$ und Diacylglycerol (DG). Diese lösen dann die notwendigen intrazellulären Vorgänge aus. Die Aktivierung der Mastzellen und Basophilen führt auf jeden Fall zu ihrer Degranulation und Freisetzung der in den Granula enthaltenen Mediatoren. Gleichzeitig werden biosynthetische Abläufe in Gang gesetzt, die Prostaglandine und Leukotriene sowie andere Substanzen produzieren. Die freigesetzten biologisch aktiven Substanzen sind dann für die klinische Manifestation der allergischen, aber auch von pseudoallergischen Reaktionen verantwortlich. Die Ähnlichkeit von allergischen und pseudoallergischen Reaktionen im Hinblick auf ihre klinischen Symptome kommt dadurch zustande, dass die pseudoallergischen Reaktionen die gleichen Mediatoren und Verstärkersysteme aktivieren wie bei den allergischen Erkrankungen. Beiden Reaktionstypen ist die Bildung von Histamin und Sulfidoleukotrienen gemeinsam. Bemerkt werden sollte, dass Histamin insbesondere durch Mastzellen und Basophile produziert wird, die Sulfidoleukotriene werden jedoch auch von stimulierten Eosinophilen, Monozyten und Endothelzellen gebildet. Der Zellantigen-
Tab.1 Testverfahren bei allergischen Reaktionen

\begin{tabular}{ll}
\hline In-vivo-Testung & In-vitro-Testung \\
\hline $\begin{array}{l}\text { Pricktestung } \\
\text { Scratchtestung }\end{array}$ & $\begin{array}{l}\text { serologische Tests } \\
\text { spezifisches IgE } \\
\text { (RAST, Magic LITE, AlaSTAT, CAP) }\end{array}$ \\
Intrakutantestung & Western Blot \\
Epikutantestung & zelluläre Tests \\
orale Provokationstestung & Histaminfreisetzungstest \\
& $\begin{array}{l}\text { Lymphozytentransformationstest } \\
\text { Zellantigenstimulationstest }\end{array}$ \\
\hline
\end{tabular}

stimulationstest ist in der Lage, beide Typen, sowohl die allergische als auch die pseudoallergische Reaktion, zu erfassen.

Die Hymenopterengiftallergie zählt zu den typischen IgE-vermittelten Reaktionen vom Soforttyp [10]. Sensibilisierte Mastzellen und Basophile besitzen an ihrer Oberfläche spezifische IgE-Antikörper. Bei Reexposition mit dem Allergen tritt eine Quervernetzung von Allergen und IgE-Antikörpern ein. Neben der Freisetzung präformierter Granula mit deren Leitsubstanz Histamin beginnt praktisch simultan die Ausschüttung neu synthetisierter Mediatoren. Ausgehend von der Arachidonsäure entstehen auf dem Cyclooxygenase-Weg Prostaglandine und auf dem Lipoxygenase-Weg die Leukotriene. Leukotriene sind also Mediatoren der Allergie vom Soforttyp.

Systemische Reaktionen auf Hymenopterenstiche beruhen in den allermeisten Fällen auf einem allergischen, anaphylaktischen Mechanismus, der ganz überwiegend IgE-vermittelt ist. Daneben kommen gelegentlich symptomatologisch gleichartige Reaktionen vor, denen offensichtlich kein IgE-vermittelter Mechanismus zugrunde liegt und deren Pathogenese zumeist unklar bleibt. Hier kann eine Auslösung durch sogenannte "short-term sensitizing anaphalactic IgG-antibodies“ oder durch eine Immunkomplexanaphylaxie diskutiert werden [17]. Auch nicht immunologische Überempfindlichkeitsreaktionen wie Idiosynkrasie, Intoleranz oder neuropsychogene Reaktionen sind in Betracht zu ziehen. Auf einer solchen Idiosynkrasiereaktion, ausgelöst durch die Histamin-freisetzende Wirkung von Hymenopterengiften, beruht möglicherweise das Auftreten von anaphylaktoiden Reaktionen nach Hymenopterenstichen bei Patienten mit Urticaria pigmentosa ohne nachweisbare Hymenopterengift-Sensibilisierung.

Die klinische Diagnostik der Hymenopterengiftallergie sollte wie bei jeder Allergie mit der Erstellung einer ausführlichen Anamnese beginnen. Sie stellt nach wie vor die verlässlichste Methode zur Bewertung allergischer Reaktionen dar. Es hat sich bewährt, den Schweregrad von systemischen, anaphylaktischen Reaktionen zu bewerten. Hierzu sind unterschiedliche Skalensysteme in Gebrauch. Häufig angewandt wird die Einteilung nach H. L. Mueller [14] oder auch die Einteilung nach Ring und Messmer [18], da sie die fortschreitende Beteiligung einzelner Organsysteme an der anaphylaktischen Reaktion dynamisch und therapierelevant beschreibt.

Der Hauttest wird mit kommerziell erhältlichem Bienen- (Apis mellifera) und Wespengift (Vespula germanica, Vespula vulgaris) vorgenommen. Es wird die Reaktionsschwelle, d.h. diejenige Konzentration, die zum Auftreten mindestens einer +-Re- 


\section{ARACHIDONSÄURE KASKADE}

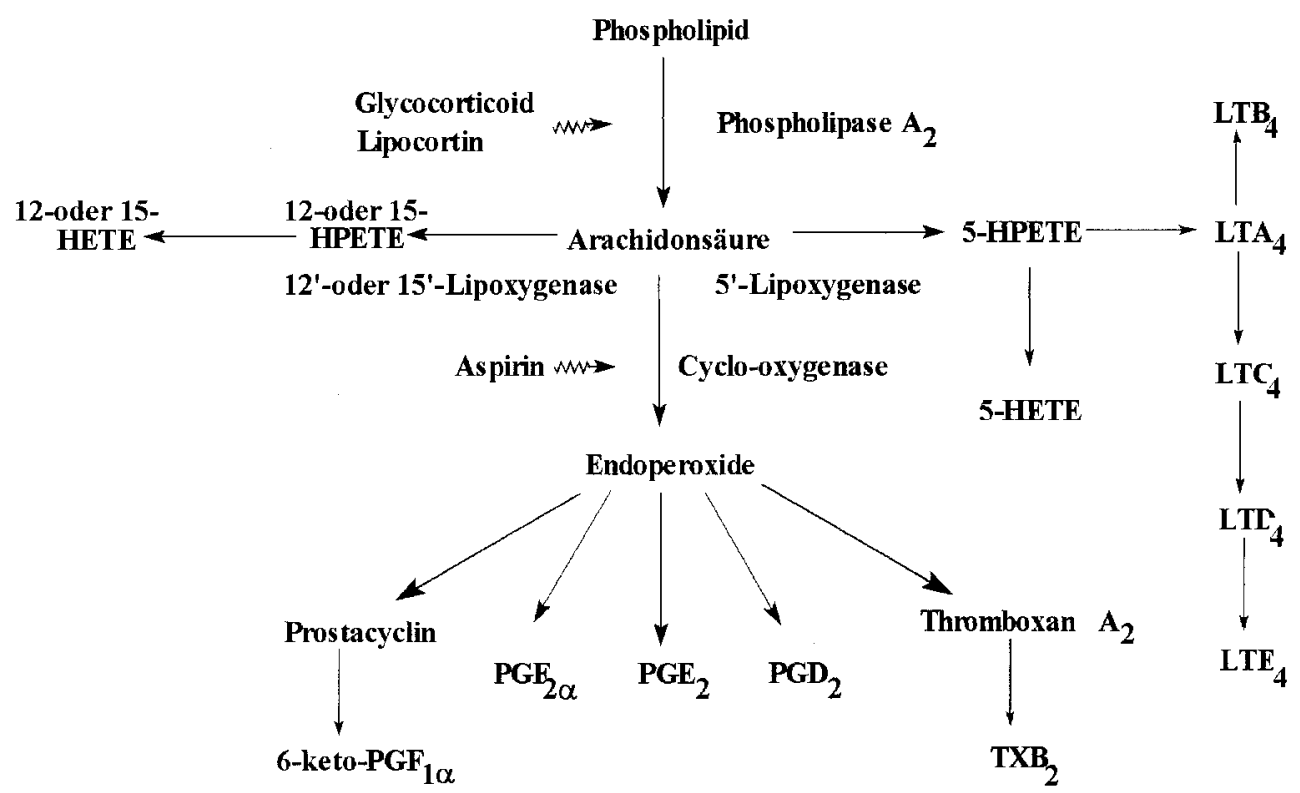

Abb. 2 Bildung von Sulfidoleukotrienen und Prostaglandinen. aktion führt, bestimmt. Bewährt hat sich der Pricktest, wobei die Konzentration schrittweise in Zehnerpotenzen von $0,01 \mu \mathrm{g} / \mathrm{mL}$ auf $300 \mu \mathrm{g} / \mathrm{mL}$ gesteigert wird. Alternativ kann intrakutan mit 100- bis 1000fach höheren Verdünnungen getestet werden. Es ist zu beachten, dass bei Toxinkonzentrationen von $100 \mu \mathrm{g} / \mathrm{mL}$ im Pricktest bzw. $0,1 \mu \mathrm{g} / \mathrm{mL}$ im Intrakutantest möglicherweise bereits durch die Giftwirkung, also nicht allergisch bedingte Hautreaktionen auftreten können.

Der Hauttest gehört nach wie vor zu den genauesten Methoden für die Ermittlung einer Insektengiftallergie; seine Sensitivität liegt zwischen $90 \%$ und $100 \%$ und die Spezifität beträgt $95-100 \%[15,22]$.

Die bereits genannten In-vitro-Methoden haben trotz der hohen Verlässlichkeit des klassischen Hauttests einige Vorteile. Sie sind nicht zuletzt für die Patienten mit einem geringeren Zeitaufwand verbunden und schließen die Gefahren allergischer Zwischenfälle bei der Testung aus.

Das Ziel der vorliegenden Arbeit sollte es sein, die diagnostische Aussagekraft der CAST-Ergebnisse im Vergleich mit anderen Routinemethoden (Hauttest, CAP) zu vergleichen und zu evaluieren. Einerseits existieren zu dieser Fragestellung bisher nur wenige Arbeiten [2,3,9] und zum anderen soll gezeigt werden, dass der CAST nicht nur als komplementäre Methode aufzufassen ist, sondern insbesondere im Rahmen der Diagnostik von Hymenopterengiftallergien als eigenständige Methode durchaus seine Berechtigung besitzt.

\section{Patienten und Methoden}

Patientengut

Es wurden 84 Patienten (35 Männer, 49 Frauen) mit Verdacht auf Insektengiftallergie mit Hilfe des CAST-ELISA-Test von Bühlmann Laboratories AG (Schweiz) untersucht. Die Altersverteilung lag zwischen 16 und 71 Jahren. Alle Patienten waren keiner Immuntherapie mit Insektengift unterzogen worden. Der Schweregrad der allergischen Soforttyp-Reaktion wurde nach den Kriterien von H. L. Mueller [14] beurteilt, wobei alle Schweregrade vertreten waren (Lokalreaktion: 6; Grad I: 23; Grad II: 38; Grad III: 8; Grad IV: 9). Als Vergleich diente eine Kontrollgruppe von 10 nicht-allergischen CAP-negativen Personen auf Bienen- und Wespengift.

\section{Methodik}

Hauttest

Der Hauttest wurde als Prick- und Intrakutantest ausgeführt, wobei als Schwellwert jene Konzentration definiert ist, die gerade noch eine positive Sofortreaktion bewirkt (Quaddeldurchmesser $\geq 5 \mathrm{~mm}$ ). Als Allergene wurden Bienengiftallergen I 1 und Wespengiftallergen I 3 (ALK, Kopenhagen, Dänemark) jeweils in Verdünnungen von $0,01 \mu \mathrm{g} / \mathrm{mL}$ bis $300 \mu \mathrm{g} / \mathrm{mL}$ für den Pricktest und von $0,001 \mu \mathrm{g} / \mathrm{mL}$ bis $1,0 \mu \mathrm{g} / \mathrm{mL}$ für den Intrakutantest verwendet. Die Negativkontrollen wurden mit NaCl-Lösung (Allergopharma, J. Ganzer KG) und die Positivkontrollen mit Histamin 1,0 $\mathrm{g} / \mathrm{mL}$ (ALK, Kopenhagen, Dänemark) durchgeführt.

\section{RAST}

Gesamt-IgE-Bestimmungen und spezifische IgE-AntikörperBestimmungen für Bienen- und Wespengifte erfolgten mit Hilfe des CAP-Systems von Kabi Pharmacia Diagnostics. Dabei wurden die Ergebnisse mit CAP-Klasse $\geq 2(\geq 0,7 \mathrm{kU} / \mathrm{L})$ als positiv bewertet.

\section{CAST}

Der Zellantigenstimulationstest (CAST) wird aus EDTA-Blut mittels Dextransedimentation durchgeführt. Jeweils $2 \mathrm{ml}$ EDTA-Blut werden mit 0,5 ml Dextran leicht geschwenkt. Anschließend wird 90 min bei Raumtemperatur inkubiert. Die 


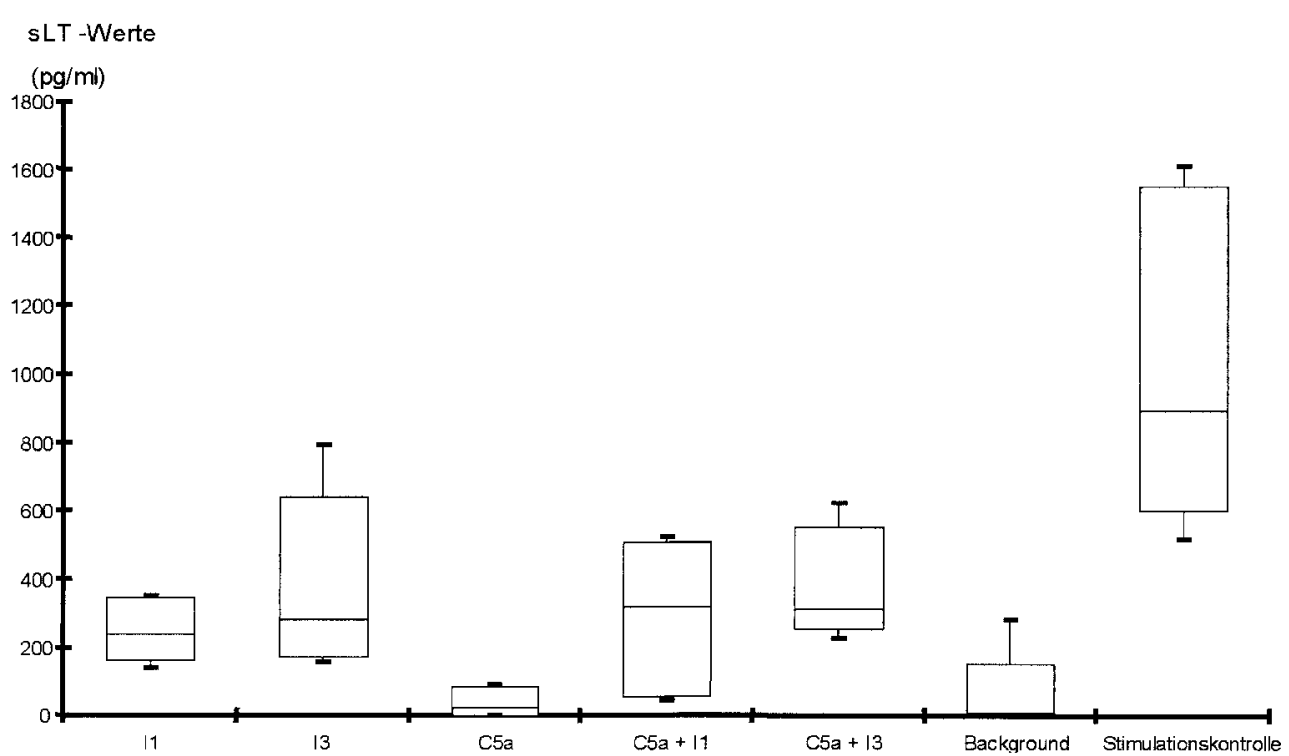

Abb. 3 Mittlere Sulfidoleukotrienwerte von 10 Probanden mit negativer Anamnese auf Bienenund Wespengifte.
Tab. 2 Korrelationen verschiedener In-vitro-Untersuchungen (CAPSystem, CAST) mit Hauttests: Biene (I 1)

\begin{tabular}{lccclc}
\hline \multicolumn{3}{c}{ Hauttest } & & \\
CAP & & & & & \\
& & + & - & Sensitivität & \\
CAP n = 84 & + & 27 & 16 & $27 / 31$ & $87 \%$ \\
& - & 4 & 37 & Spezifität & \\
& & & & $37 / 53$ & $70 \%$ \\
CAST & & & & & \\
& & + & - & Sensitivität & \\
CAST n = 84 & + & 27 & 18 & $27 / 29$ & $93 \%$ \\
& - & 2 & 37 & Spezifität & \\
& & & & $37 / 55$ & $67 \%$ \\
\hline
\end{tabular}

Tab. 3 Korrelationen verschiedener In-vitro-Untersuchungen (CAPSystem, CAST) mit Hauttests: Wespe (I 3)

\begin{tabular}{|c|c|c|c|c|c|}
\hline & & Haut & & & \\
\hline \multicolumn{6}{|l|}{ CAP } \\
\hline & & + & - & Sensitivität & \\
\hline \multirow[t]{3}{*}{ CAP $n=84$} & + & 71 & 3 & $71 / 77$ & $92 \%$ \\
\hline & - & 6 & 4 & Spezifität & \\
\hline & & & & $4 / 7$ & $57 \%$ \\
\hline \multicolumn{6}{|l|}{ CAST } \\
\hline & & + & - & Sensitivität & \\
\hline \multirow[t]{3}{*}{ CAST $n=84$} & + & 73 & 3 & $73 / 76$ & $96 \%$ \\
\hline & - & 3 & 5 & Spezifität & \\
\hline & & & & $3 / 8$ & $63 \%$ \\
\hline
\end{tabular}

obere Phase wird mit einer Transferpipette in ein Zentrifugenröhrchen überführt und bei $130 \times \mathrm{g} 15 \mathrm{~min}$ bei $4{ }^{\circ} \mathrm{C}$ zentrifugiert. Der Überstand wird anschließend verworfen und das Zellpellet in $2 \mathrm{ml}$ Inkubationspuffer mit $270 \mu$ Interleukin-3 aufgenommen. Anschließend gibt man $200 \mu$ Zellsuspension in Eppendorf-Tubes und führt die Stimulation in Doppelbestimmung mit $50 \mu \mathrm{l}$ Inkubationspuffer und Interleukin-3 $(200 \mu \mathrm{l}$ IP $+3 \mu \mathrm{l}$ IL-3) durch. Dabei erhält man den Background-Wert. Zwei weitere Eppendorf-Tubes mit jeweils $200 \mu \mathrm{l}$ Zellsuspension werden mit $50 \mu \mathrm{l}$ monoklonalen AntiIgE-Antikörpern (Le 27) oder mit dem monoklonalen Antikörper des hochaffinen IgE-Rezeptors stimuliert. Nach der Zahl der Allergene gibt man dann jeweils $50 \mu$ l Allergenlösung $\mathrm{zu}$ $200 \mu \mathrm{l}$ Zellsuspension. Die Stimulation wird $40 \mathrm{~min}$ bei $37^{\circ} \mathrm{C}$ durchgeführt. Danach wird bei den oben angegebenen Bedingungen 5 min zentrifugiert. Den Zellüberstand friert man bei $-20^{\circ} \mathrm{C}$ ein und führt innerhalb von 4 Wochen den Enzymimmunoassay durch.

Der CAST-Enzym-gebundene Immunosorbent Assay (ELISA) basiert auf der Tatsache, dass ein einfacher monoklonaler Antikörper nicht nur Leukotrien C4, sondern auch seine zwei Metaboliten (Leukotrien D4 und Leukotrien E4) mit gleicher Sensitivität und Spezifität erkennt [3,19]. Mit Hilfe des CAST-ELISA kann man alle Sulfidoleukotriene messen, die durch Zellstimulation bei Allergeneinwirkung produziert werden.

\section{Sensitivität und Spezifität}

Der Vergleich erfolgte unter Verwendung der 4-Felder-Tafel, wobei die Übereinstimmung mit dem Hauttest geprüft wird. Daraus ergeben sich Untergruppen, in denen die Ergebnisse übereinstimmen $(+/+=\mathrm{a}$ und $-/-=\mathrm{d})$ und solche, in denen $\mathrm{Ab}-$ weichungen auftreten $(+/-=$ c bzw. $-/+=b)$. Sensitivität und Spezifität können nach folgenden Formeln berechnet werden: Sensitivität $=100 \mathrm{a} /(\mathrm{a}+\mathrm{c})$ und Spezifität $=100 \mathrm{~d} /(\mathrm{b}+\mathrm{d})$. 

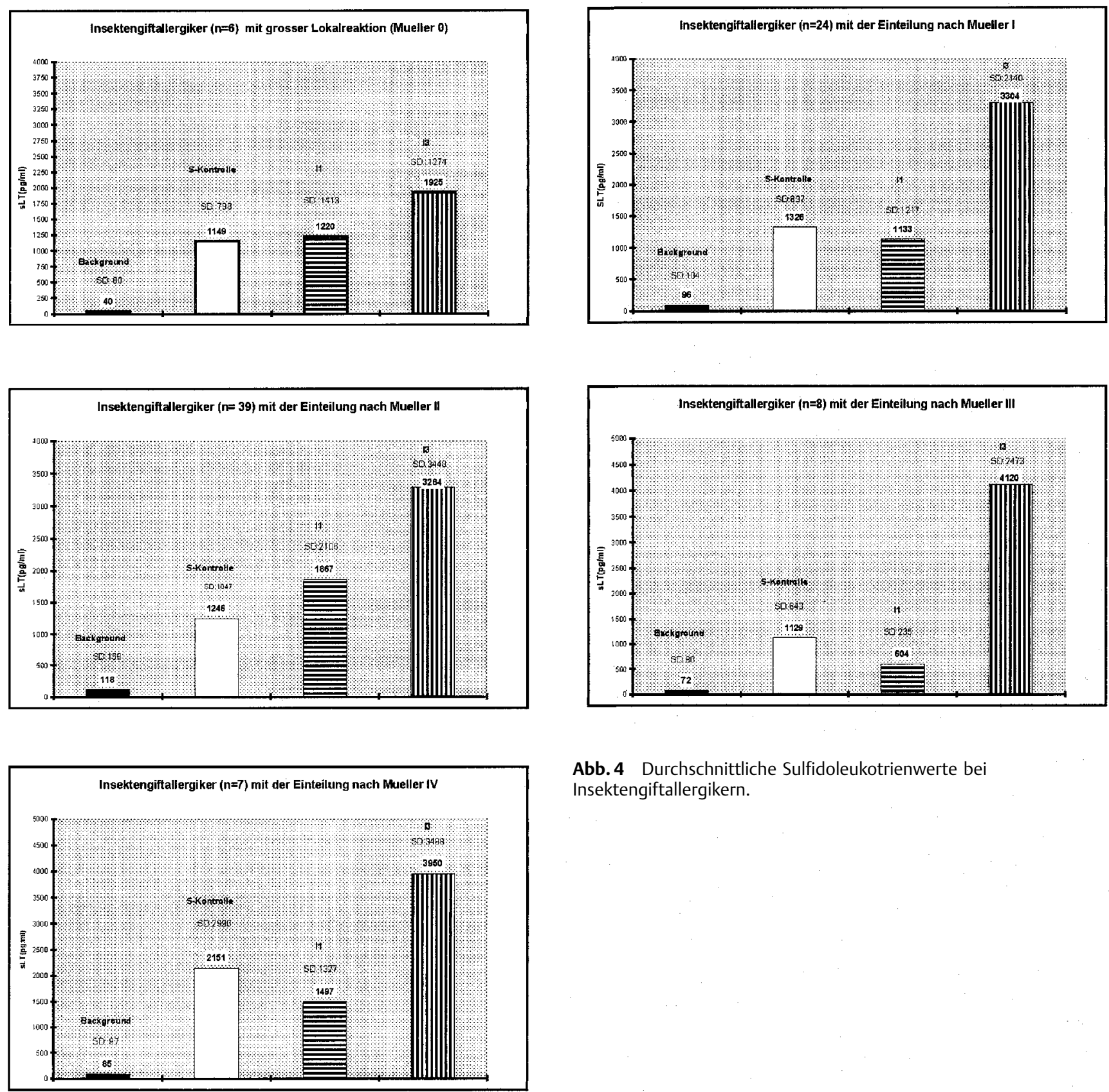

Abb. 4 Durchschnittliche Sulfidoleukotrienwerte bei Insektengiftallergikern.

\section{Ergebnisse}

Entsprechend der Vorgaben des Herstellers der Allergene wurden zunächst die Normwerte für den CAST ermittelt (Abb. 3). Dazu wurden die gemittelten Werte der Kontrollgruppe von 10 Probanden mit negativer Anamnese auf Bienen- und Wespengifte verwendet. Die mittleren Leukotrienspiegel ergeben Werte zwischen 100 und 500 pg/mL. Das Festlegen physiologischer und pathologischer Richtwerte erweist sich insbesondere wegen der über einen längeren Zeitraum schwankenden Vorgaben der Hersteller als etwas problematisch.

Die Abb. 4 zeigt die durchschnittlichen Leukotrienwerte im CAST nach Stimulation mit Bienen- und Wespengiften bei jeweils einer Allergenkonzentration von $0,2 \mu \mathrm{g} / \mathrm{mL}$ in dem von

uns untersuchten Kollektiv von Bienen- und Wespengiftallergikern. In der Darstellung wurde dabei versucht, eine Korrelation der gemessenen Sulfidoleukotrienwerte mit den in der Anamnese ermittelten Schweregraden nach Mueller herzustellen. Es zeigte sich jedoch, dass zwischen den Schweregraden und den sLT-Werten keine direkte Proportionalität besteht.

Für die Bewertung des CAST als Testmethode zur Aufklärung von Hymenopterengiftallergien wurden vergleichende Betrachtungen des CAST mit den Hauttests und den CAP-Ergebnissen vorgenommen. Dazu wurden 4-Felder-Tafeln verwendet, aus denen Sensitivität und Spezifität berechnet werden können. Wie aus Tab. 2 und $\mathbf{3}$ ersichtlich ist, berechnen sich für Bienengift eine Sensitivität von 93\% und eine Spezifität 


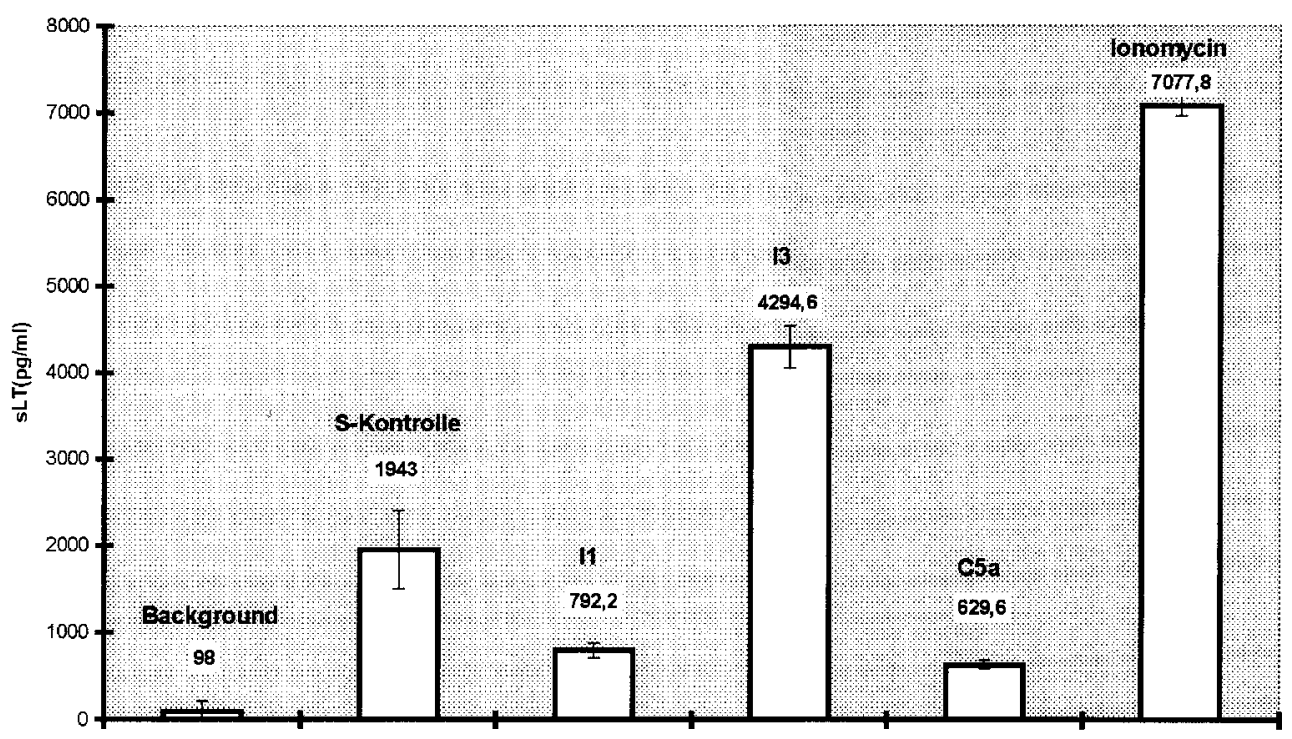

Abb. 5 Intraassay eines Patienten im Zellantigenstimulationstest.

Tab. 4 Zellantigenstimulationstest (Bienen- und Wespengiftallergie)

\begin{tabular}{|c|c|c|c|c|c|}
\hline Patient & $\begin{array}{l}\text { spezif. IgE-AK- } \\
\text { Klasse (CAP) }\end{array}$ & $\begin{array}{l}\text { Background } \\
(\mathrm{pg} / \mathrm{mL})\end{array}$ & $\begin{array}{l}\text { Stimulationskontrolle } \\
(\mathrm{pg} / \mathrm{mL})\end{array}$ & $\begin{array}{l}\text { Allergenstimulation } \\
(\mathrm{pg} / \mathrm{mL})\end{array}$ & Hauttest \\
\hline \multirow[t]{5}{*}{1} & I $1=0$ & 82 & 1555 & 645 & neg. \\
\hline & $13=0$ & & & 1859 & neg. \\
\hline & & & & $C 5 a=288$ & \\
\hline & & & & $C 5 a+I 1=2223$ & \\
\hline & & & & $C 5 a+13=1602$ & pseudoallergische Reaktion \\
\hline \multirow[t]{3}{*}{2} & $\mid 1=0$ & 276 & 1972 & 694 & neg. \\
\hline & $13=4$ & & & 4563 & pos. \\
\hline & & & & $C 5 a=663$ & $\begin{array}{l}\text { V.a. Mitbeteiligung einer } \\
\text { pseudoallergischen Reaktion }\end{array}$ \\
\hline \multirow[t]{3}{*}{3} & $11=4$ & 106 & 1900 & 7057 & pos. \\
\hline & $13=3$ & & & 5296 & neg. \\
\hline & & & & $C 5 a=670$ & $\begin{array}{l}\text { V.a. Mitbeteiligung einer } \\
\text { pseudoallergischen Reaktion }\end{array}$ \\
\hline
\end{tabular}

von $67 \%$, für Wespengift eine Sensitivität von $96 \%$ und eine Spezifität von $63 \%$.

Im Vergleich zu den gleichzeitig ermittelten CAP-Werten zeigte sich im Rahmen unserer Untersuchungen, dass die Resultate des CAST günstiger ausfallen.

In Abb. 5 wird der Intraassay $(n=5)$ eines Patienten im Zellantigenstimulationstest gezeigt. Dazu wurden Untersuchungen verschiedener Serumproben des gleichen Probanden im Verlauf eines Tages durchgeführt. Diese Ergebnisse zeigen die sehr gute Reproduzierbarkeit des CAST.

In einigen Fällen wurden zusätzliche Stimulationen der Zellen mit dem Anaphylatoxin C 5a vorgenommen. Die entsprechenden Ergebnisse sind in Tab. 4 dargestellt.

Damit war es möglich, in einem Fall das Vorliegen einer pseudoallergischen Reaktion nachzuweisen und in zwei weiteren
Fällen den Verdacht auf die Mitbeteiligung einer pseudoallergischen Reaktion wahrscheinlich zu machen.

\section{Diskussion}

Mit der Etablierung des Zellantigenstimulationstests ergeben sich neue Möglichkeiten im Bereich der zellulären In-vitroDiagnostik für allergische und auch pseudoallergische Reaktionen auf Insektengifte und, wie wir auch in früheren Arbeiten zeigen konnten, gegenüber Arzneimitteln und Nahrungsmitteln $[7,8]$.

In der vorliegenden Arbeit konnte bei 83 Patienten mit Verdacht auf Hymenopterengiftallergie der Nachweis dieser Allergien mit Hilfe des CAST erbracht und quantifiziert werden. Die gemessenen Sulfidoleukotrienwerte stehen in Übereinstimmung mit den Schweregraden nach Mueller, wobei allerdings zwar eine direkte, aber keine proportionale Korrelation zwi- 
schen den Absolutbeträgen der Sulfidoleukotriene und den Mueller-Graden besteht.

Im Vergleich zum Hauttest zeigt sich eine signifikante Übereinstimmung. Die Sensitivitäten und Spezifitäten von 93/70\% für Bienengift und von 96/63\% für Wespengift sind deutlich höher als bei vergleichbaren Arbeiten zu dieser Problematik und machen den CAST nicht nur zu einer Referenzmethode zur Ergänzung des Hauttests, sondern zu einem eigenständigen In-vitro-Testverfahren mit guter Reproduzierbarkeit und Genauigkeit. In unseren Untersuchungen weist der CAST im Vergleich zum CAP auch eine bessere Übereinstimmung mit dem Hauttest auf. Die gute Reproduzierbarkeit des CAST konnte durch eine Intraassay-Variation gezeigt werden.

Als besonders geeignet erweist sich der CAST bei der Aufklärung und dem Nachweis von pseudoallergischen Reaktionen. Unter einer pseudoallergischen Reaktion oder Intoleranz versteht man Überempfindlichkeitsreaktionen, deren klinische Symptomatik der einer Allergie ähnelt, ohne dass eine immunologische Reaktion vorliegt. Schlumberger [20], der den Begriff der Pseudoallergie prägte, weist darauf hin, dass Arzneimittel und andere chemische Substanzen auch Wechselwirkungen mit den Effektor- und Verstärkersystemen eingehen können, die eigentlich nur durch spezifische Immunmechanismen aktiviert werden. Neuere Arbeiten haben gezeigt, dass Anaphylatoxine, insbesondere das C 5a, über einen IgE-unabhängigen Mechanismus auf die Zielzelle wirken [12]. Eine IgE-unabhängige Degranulation durch entstehendes C 5a von basophilen Granulozyten kann unter verschiedenen Bedingungen initiiert werden [1]. Dazu gehören einmal von Antikörpern unabhängige, pseudoallergische Reaktionen, das Fehlen von Inaktivatoren oder Regulatoren unter pathologischen Bedingungen und eine AgAK-Komplex-vermittelte Histaminfreisetzung durch Aktivierung des Komplementsystems (Typ III) sowie auch eine über homozytrope AK der IgG-Klasse vermittelte Histaminfreisetzung (Typ II der allergischen Reaktion).

Aus diesen Überlegungen heraus führt man den Zellantigenstimulationstest bei Verdacht auf eine pseudoallergische Reaktion mit C 5a durch. Nach den Arbeiten von Czech et al. [4] ist eine Inkubation mit C $5 \mathrm{a}$ hilfreich bei der Aufklärung von pseudoallergischen Reaktionen gegenüber von Acetylsalicylsäure. In unseren früheren Arbeiten konnten durch Inkubation mit $C$ 5a Intoleranzreaktionen auf Arzneimittel nachgewiesen werden [8].

Auch bei der Untersuchung der Hymenopterengiftallergien erbrachte die zusätzliche Stimulation mit dem Anaphylatoxin C 5 a den Nachweis über das Vorliegen einer pseudoallergischen Reaktion bzw. den Hinweis auf eine Beteiligung von Intoleranzreaktionen. Gerade unter diesem Aspekt und auch aufgrund der guten Sensitivitäten und Spezifitäten des CAST im Vergleich zum Hauttest und den CAP-Ergebnissen halten wir den Zellantigenstimulationstest für eine sehr geeignete Testmethode in der Diagnostik von Hymenopterengiftallergien.

\section{Literatur}

${ }^{1}$ Bischoff SC, Kurimoto Y, Dahinden CA. Interleukin-3 and Granulocyte/Macrophage Colony-Stimulating Factor Change the Anaphylatoxin-Induced Basophil Mediator Release Pattern. In: Ring J, Przybilla B. New Trends in Allergy III. Springer, 1991: 161 - 165
${ }^{2}$ Cahen YD, Maly FE, Wüthrich B. Cellular Antigen Stimulation Test (CAST) - Verwendbarkeit in der Diagnostik von Insektengiftallergien. Schweiz Med Wochenschr 1997; 127: 5-11

${ }^{3}$ CAST Brochure. Allschwil, Switzerland: Bühlmann Laboratories, 1993

${ }^{4}$ Czech W, Schöpf E, Kapp A. Granulocyte Mediator Release in Pseudoallergic Reactions. In: Ring J, Przybilla B. New Trends in Allergy III. Springer, 1991: 310-317

${ }^{5}$ De Weck AL, Furukawa K, Urwyler A, Stadler BM, Dahinden CA, Maly FE. Sulfidoleukotriene - ELISA Assay: A New Cellular Approach To The Diagnostic of Allergies. Poster presented at EAACI. Paris: May 1992

${ }^{6}$ De Weck AL, Stadler BM, Urwyler A, Wehner HU, Bühlmann RP. Cellular Allergen Stimulation Test - A New Dimension in Allergy Diagnostics. ACI News 1993; 5: 9-14

${ }^{7}$ Hipler UC. Vergleich von drei Testsystemen zur quantitativen Gesamt-IgE-Bestimmung bei Patienten mit atopischer Dermatitis. Lab med 1993; 17: 400

${ }^{8}$ Hipler UC. Erfahrungen mit dem CAST in der Diagnostik von Allergien und Intoleranzreaktionen gegenüber Arzneimitteln. Der Deutsch Dermat 1997; 3: 236-252

${ }^{9}$ Höxtermann S, Auer T, Altmeyer P. Zelluläre In-vitro-Diagnostik mittels CAST-ELISA: Leukotriennachweis bei Wespengiftallergie. Allergologie 1995; 18: 287-291

${ }^{10}$ Jarisch R. Die Bienengiftallergie. Modell einer IgE mediierten Soforttypallergie. Wien Klin Wochenschr 1980; 12: 92

11 Jäger L. Im Brennpunkt: Stellenwert der In-vitro-Diagnostik bei allergischen Erkrankungen. Allergologie 1995; 18: 114-117

12 Jürgensen $H$, Behrendt $H$, Schmutzler W. Über die Bedeutung der Anaphylatoxine im allergischen und entzündlichen Geschehen. Allergologie 1990; 13: 120-127

${ }^{13}$ Kurimoto Y, De Weck AL, Dahinden CA. The effect of interleukin 3 upon IgE-dependent and IgE-independent basophil degranulation and leukotriene generation. Eur J Immunol 1991; 21: 361 368

${ }^{14}$ Mueller HL. Diagnosis and treatment of insect sensitivity. J Asthma Res 1986; 3: 331 - 333

${ }^{15}$ Müller UR. Insect sting allergy: clinical pictures, diagnosis and treatment. Stuttgart: Gustav Fischer, 1990: 76

${ }^{16}$ Przybilla B. Bienen- und Wespengiftallergie. Hautarzt 1993; 44: $611-623$

17 Przybilla B, Ring J. Diagnostik und Therapie der Allergie vom Sofort-Typ gegenüber Bienen- und Wespengift. Allergologie 1985; 8: $31-39$

18 Ring J, Messmer K. Incidence and severity of anaphylactoid reactions to colloid volume substitutes. Lancet 1977; 1: 466-469

${ }^{19}$ Reinke M, Hoppe U, Thomas R, Bestmann HJ, Mollenhauer J, Brune K. A monoclonal antibody against the sulfidopeptide leukotrienes $\mathrm{LTC}_{4}$, $\mathrm{LTD}_{4}$ and $\mathrm{LTE}_{4}$. Biochemica et Biophysica Acta 1991; 1081: $274-278$

${ }^{20}$ Schlumberger HD. Pseudoallergische Reaktionen durch Arzneimittel und Chemikalien. Allergologie 1982; 5: 183-189

${ }^{21}$ Subcommittee on Insect Venom Allergy of the European Academy of Allergology and Clinical Immunology. Immunotherapy with hymenoptera venoms. Allergy 1993; 48: $36-46$

${ }^{22}$ Wüthrich B, Wick H, Crass B, Wyss S. Zur Diagnostik der Hymenopterenstich-Allergie: ein Vergleich zwischen Anamnese, Hauttesten und IgE-Bestimmungen (RAST) mit Giftextrakten. Schweiz Rundsch Med Prax 1981; 21: 934-943

\section{Dr. rer. nat. Uta-Christina Hipler}

Klinik für Hautkrankheiten Klinikum der Friedrich-Schiller-Universität Jena Erfurter Straße 35

07740 Jena 